\title{
Las memorias del miedo y el miedo a las memorias. Aproximación a la Guerra Civil española y sus herencias en comunidades rurales del sur de Aragón
}

\author{
María Alexia Sanz Hernández \\ Universidad de Zaragoza. Facultad de Ciencias Sociales y Humanas. \\ Departamento de Psicología y Sociología \\ alexsanz@unizar.es
}

\section{Resumen}

A partir de una perspectiva etnometodológica, y fundamentalmente a través del análisis del discurso de informantes, el artículo propone, por una parte, un análisis de las principales dimensiones temáticas manifiestas en las narrativas que, desde los recuerdos individuales, configuran la memoria del miedo que se entreteje en comunidades rurales del sur de Aragón, a propósito de la vivencia de la Guerra Civil española. Al mismo tiempo, analiza las principales expresiones del miedo a esa memoria, indagando en sus interrelaciones conflictivas con la articulación de un discurso histórico, oficial y público sobre este acontecimiento. El miedo a la muerte, al hambre y a la destrucción, así como la huida o el ocultamiento, son los ejes principales de unos relatos sobre el riesgo asociados al hecho bélico; mientras que el miedo a la memoria se presenta, al mismo tiempo, como una estrategia de reconstrucción de lo comunitario en el escenario de la posguerra, secuela del miedo inherente a los sucesos vivenciados y en tanto que recurso para ocultar valoraciones del presente a la luz del pasado. El miedo es, por tanto, objeto y sujeto de la memoria asociada a este hecho histórico, y esa dualidad se encuentra en una interrelación permanente, que perdura en el tiempo, con todas las complejidades asociadas a esa circunstancia, sintetizada en la perdurabilidad, hasta el presente, de la memoria del miedo (pasado) y el miedo (de lo) instituido a la memoria (instituyente).

Palabras clave: política; historia; oralidad; represión; comunidad; posguerra; riesgo; conflictos bélicos.

Abstract. Memories of fear and fear of memories: An approach to the Spanish Civil War and its legacy in rural communities of Southern Aragon

By applying an ethnomethodological research perspective, and through the discourse analysis of individuals interviewed in rural communities of Southern Aragon, this article aims to analyse the main thematic dimensions of the narratives based on individual remembrances 
that configure the memory of fear surrounding the Spanish Civil War. At the same time, it examines the core expression of the fear of this memory by investigating the conflicting interrelationships between memory and the official and public historical discourse on this event. The main themes of the discourses on the risks associated with the war are death, hunger, and destruction, as well as the need to flee or hide from the enemy. The fear of memory is, at the same time, a post-war strategy for the reconstruction of the sense of community, a consequence of the war experience, and a resource for concealing opinions on current events based on past ones. Fear is thus both an object and subject of the memory of the Spanish Civil War; a duality that could be summarised in the complexities associated to the continuity, until the present, of the memory of (past) fear and the fear of (instituting) memory.

Keywords: politics; risk; history; orality; repression; community; post-war; armed conflict.

\author{
Sumario \\ 1. Introducción 4. Conclusiones: la memoria del miedo \\ y el miedo a la memoria \\ 2. Recuerdos de la (pos)guerra: \\ Referencias bibliográficas
}

3. El miedo a la memoria: silencios y valoraciones

\title{
1. Introducción
}

La configuración de la historia descansa, en tanto que proceso, sobre un éxito o un fracaso significativo de los individuos que conviven en una sociedad o en un grupo social organizado de forma duradera. La memoria colectiva, de la cual se alimenta ese proceso de configuración de la historia, cobra cuerpo en tramas narrativas. Los vínculos entre la historia — como relato oficial del pasado - y la memoria — como narrativa cotidiana, también de los acontecimientos pretéritos- son complejos y, con frecuencia, conflictivos. Y esta conflictividad se acrecienta en el caso de aquellos hechos que, en sí mismos, también aparecen marcados por esta condición, como pueden ser las guerras, en tanto que expresión más extrema del conflicto social.

En este artículo, tomamos como eje analítico esta segunda dimensión de lectura del pasado, más anclada a las tramas de la vida cotidiana de los sujetos y las comunidades, a sus flujos y devenires, que a la escritura permanente de los libros de historia. La historia, en tanto que discurso del (y desde el) poder, aparece intrínsecamente asociada, en la modernidad, a su institución central, el estado. Es un relato fijo, marcado por el (deseo de) estatismo, de devenir verdad absoluta y eterna, y es una de las expresiones más evidentes de los vínculos entre saber y poder (Foucault, 1979). La memoria, por el contrario, se entrelaza al movimiento y la multiplicidad propios de la vida cotidiana, en un proceso activo y creativo, de reinvención permanente. Cobra cuerpo, por consiguiente, en unas narrativas más dinámicas y flexibles, por tanto, y más esquivas, también. 
Desde la memoria, nos aproximamos a un hecho particularmente conflictivo - el que más, sin duda - en la historia de España del siglo pasado: la Guerra Civil española (1936-1939). Se trata, por tanto, de una indagación atravesada por una doble conflictividad: de un lado, la intrínseca al hecho en torno al cual se articula la memoria; del otro, la singular tensión que, en el caso de tal acontecimiento, se pone de manifiesto entre los relatos de la historia y las tramas narrativas de la memoria. El cisma que supuso en la sociedad española ese hecho y la prolongación en el tiempo de su herencia, en forma de una dictadura, marcan la singular conflictividad de la configuración de los imaginarios acerca del mismo, de sus lecturas e interpretaciones, más de siete décadas después de haber finalizado.

Dadas estas particularidades, la memoria social se presenta como dimensión analítica pertinente para la indagación en torno a las configuraciones del universo simbólico-interpretativo sobre tal hecho. Una cuestión tan especialmente polémica encuentra, en los resortes de la memoria, un correlato que justamente permite, en toda su extensión y complejidad, juegos de reinterpretación permanentes y abiertos, en correspondencia con ese planteamiento constante de nuevos interrogantes concernientes a los procesos del olvido, la distorsión y la reconstrucción positiva o negativa del pasado que supone la memoria, como recurso de articulación de narrativas (Halbwachs, 1968).

Asumir la perspectiva de la memoria para acercarse a las narrativas sobre la Guerra Civil española también ofrece otras dos condiciones apropiadas para el análisis que se propone. En primer lugar, porque la memoria se encuentra en un estrecho y constante entrelazamiento con el presente, de manera que los cambios en el mundo que proyecta la narración en sus estructuras, realidades simbólicas y dimensiones temporales exigen, una y otra vez, retoques en las narraciones para readaptarlas a los nuevos horizontes de la experiencia (Sanz y Ramos, 2009).

En segundo lugar, debemos tener en cuenta, también, la peculiar articulación entre lo individual y lo colectivo, que atraviesa los procesos de emergencia y reinvención constante de la memoria. Los recuerdos individuales confluyen, en el tiempo, en un proceso de recreación continuo de sistematización y estructuración que son el soporte de los vínculos del individuo con la comunidad. Los recuerdos, de manera inherente, tienen un carácter personal, pues se basan en la memoria como facultad psíquica individual, pero, de su sedimentación e innovación, su ordenamiento y estructuración, emerge una dimensión de orden superior y diferente - la memoria social —, que adopta formas y manifestaciones diversas, en correspondencia con los grupos que las crean, las mantienen, las transmiten y las transforman en el espacio y en el tiempo.

La memoria, en un juego de superación de la capacidad mental individual, deviene social y se transforma en colectiva cuando es compartida por los miembros de una comunidad determinada, que le dan contenido y forma y la explicitan en narraciones, símbolos y rituales. Esta dualidad de la memoria — su inherente naturaleza social, a la vez que su sustrato último individual — la convierten en una dimensión particularmente interesante para la indagación 
en las interrelaciones entre lo individual y lo social, cuestión central y - si se quiere- dimensión de última instancia en todas las teorías sociológicas, desde las escuelas clásicas hasta los enfoques contemporáneos (Bilbao, 2007).

Esta tensa dualidad se presenta con mayor énfasis sobre todo en el caso de la memoria colectiva en torno a un hecho tan conflictivo como es la guerra, tan personal y, al mismo tiempo, de tanto significado social e histórico. Circunstancia que se ve reforzada cuando se trata, además, de una contienda de carácter civil, expresión de un conflicto en el interior de una de esas comunidades imaginadas modernas, en términos de Anderson (1993), que es un estado nación. La dimensión extrema de ese tipo de enfrentamiento armado, como recuerda Beevor (2009), quedó magistralmente descrito por Antoine de Saint-Exupéry, cuando escribía: «Una guerra civil no es una guerra, sino una enfermedad. El enemigo es interior. Lucha uno casi contra sí mismo». Esta imagen de un enfrentamiento bélico contra la mismidad que supone el que ambas partes sean miembros de una misma comunidad, en este caso un estado nación, supone no solo una particularidad para el conflicto en sí mismo, sino también para la memoria sobre este.

La flexibilidad y la movilidad, ese proceso de reinvención cotidiana que la caracteriza, ha hecho precisamente que la memoria haya sido una atalaya investigativa desde la cual se ha indagado con frecuencia en la Guerra Civil española (Aguilar Fernández, 1996; Mangini González, 1997; Luengo, 2004; Fraser, 2005). De igual forma, algunas lecturas de la conflictividad propia de ese episodio bélico lo han asociado no al enfrentamiento entre izquierda y derecha, sino a un conflicto con dos ejes que, precisamente, también se articulan en las tensiones que atraviesan la configuración de las narrativas de la memoria. De una parte, el centralismo estatal contra la independencia regional y, de la otra, el autoritarismo contra la libertad del individuo (Beevor, 2009: 7-8). Como vemos, las dos cuestiones a que hacen referencia estos dos ejes forman parte de la emergencia de la memoria: por un lado, el desafío al estado, a través de su forma discursiva hegemónica instituida, el relato de la historia; por el otro, sus ya señalados sustrato intrínsecamente individual y, al mismo tiempo, su naturaleza inherentemente social.

Para la indagación en la memoria sobre la Guerra Civil, hemos tomado como actores discursivos a habitantes de zonas rurales del sur de Aragón, donde la contienda tuvo episodios sangrientos y dramáticos ${ }^{1}$. La selección es pertinente para la indagación que se propone, en primer lugar, por la importancia específica de este territorio en procesos históricos significativos del conflicto español: la resistencia republicana al levantamiento militar (experiencias colectivizadoras, presencia miliciana), la batalla de Teruel (invierno de 1937 a 1938), el repliegue ordenado y eficaz de las tropas para la defensa de Valencia (las batallas de Sarrión, Albentosa y Javalambre en julio de 1938), la evacuación de la población

1. El presente artículo parte del trabajo de campo iniciado en 2009 y financiado por el Gobierno de Aragón en el marco de las acciones de apoyo a proyectos de investigación, en su convocatoria de 2008 y dentro del Programa Amarga Memoria. 
ante el avance de las tropas sublevadas, la agonía de las tropas republicanas y su rendición al final de la guerra y, tras esta, la otra gran guerra silenciosa librada en los escenarios rurales españoles: la resistencia armada de los guerrilleros (11 a Sector del AGLA, Agrupación Guerrillera de Levante y Aragón).

En segundo lugar, la selección es relevante por los impactos del propio conflicto que se testimonian desde estas comunidades (ciertamente, con rasgos que muestran gran similitud a lo vivenciado y/o recordado en otras zonas rurales, pero con interesantes particularidades): los procesos de construcción del enemigo (bases políticas del miedo), la revolución en la cotidianidad, las estrategias para soportar la cercanía de la muerte, la cotidianización de la guerra, la carestía y la enfermedad, la negación del duelo o los procesos forzados de sostenimiento de los vínculos de sociabilidad en una cultura de sobrevigilancia, represión y silencios forzados.

Tomar lo local como escenario para la indagación en la memoria, en tiempos de una ampliación y virtualización de lo comunitario a través de los usos sociales de las tecnologías de la información y la comunicación (TIC), también resulta especialmente significativo. Se trata, en este caso, no de comunidades ancladas en relaciones sociales sin copresencia, sino en vínculos sociales con copresencia (Sotolongo y Delgado, 2006), configuradas en la interacción personal y en unas relaciones comunicativas básicamente interpersonales, en unas dinámicas presenciales de intersubjetividad. En el contexto de lo que Huyssen (2002) ha denominado «memory boom» y ante la inherente conflictividad de los procesos de configuración de la memoria, este anclaje en lo local ofrece la posibilidad de un análisis más profundo de esa encrucijada que apunta este autor entre el pasado mítico y el pasado real, pues solo la perspectiva local, en el trabajo empírico sobre la memoria, puede permitirnos profundizar en determinados procesos, comprensibles solo a partir del análisis de los flujos relacionales entre informantes en su contexto comunitario inmediato (en este caso, el pueblo).

A ello se agrega la reconocida importancia de la oralidad en el entorno rural, en tanto que recurso de continuidad y entrelazamiento intergeneracional, de transmisión de tradiciones y costumbres, medio imprescindible para la articulación y la permanencia de lo comunitario local (Boito y De la Cruz, 2000). Ese rol central de la oralidad en el escenario rural es compartido también por memoria, que se configura, emerge, se reinventa y perdura, en gran medida, a través de narrativas precisamente orales, aunque también se nutre de la visualidad, los rituales y los símbolos. En este entorno, los procesos de configuración de la memoria colectiva se mantienen mucho más anclados — siguiendo el análisis temporal que propone Ibáñez (1988)— a ese mecanismo de sedimentación que es la relación entre sujeto y sujeto, y toma como soporte el propio cuerpo, antes que la expresión escrita o, incluso más allá, en las bases de datos. El escenario rural, más próximo a lo arcaico en términos de Maffesoli (2009), permite acceder de manera más expedita a ese entramado narrativo por medio del cual los recuerdos individuales se entretejen en la memoria colectiva. 
En este caso, nos centraremos — desde una perspectiva etnometodológicaen el análisis de los discursos que forman parte de esas narrativas configurativas de la memoria sobre la guerra, a las cuales se ha accedido a través de entrevistas en profundidad a sujetos habitantes de diez pueblos de la provincia de Teruel, ubicados en la cara sur del Pico de Javalambre, especialmente de los municipios de Manzanera, Arcos de las Salinas, Torrijas y $\mathrm{Olba}^{2}$. El acercamiento hermenéutico a los relatos de los informantes ha supuesto, en este caso, un especial desafío metodológico, al ser una temática que atrapa, implica y sumerge en mundos únicos al investigador.

Desde un punto de vista metodológico, la indagación se ha fundamentado en el juego, desde el presente, con dos planos de articulación de la narración del pasado: los recuerdos individuales en tanto que vínculos inmediatos y directos, junto a las versiones narrativas cristalizadas tras décadas de consenso y disenso comunitario. Pero, al mismo tiempo, también se ha articulado un juego dual de temporalidades: a la vez que una indagación en el pasado desde el presente, ha emergido una mirada al presente desde el pasado y sus silencios, como parte inherente de la afectividad y la efectividad de los recuerdos y de la memoria, de estas evocaciones desordenadas, condicionadas, en ocasiones exageradas y a menudo irónicas, en torno a circunstancias y sucesos generadores de miedo acontecidos durante las décadas de 1930 y 1940.

\section{Recuerdos de la (pos)guerra: la memoria del miedo}

El miedo es, en primer lugar, contenido de la memoria. No es gratuito ese carácter transversal del miedo en los recuerdos en torno a un suceso bélico y su período posterior, sobre todo porque resulta, en este caso en particular, no uno cualquiera, sino el que pudiera calificarse como el momento más tenebroso del siglo español de las tinieblas: la Guerra Civil. De hecho, como señala este autor, existe una importante tradición en los análisis de las vivencias del miedo y el terror en las indagaciones historiográficas de los últimos treinta años en España, quizás como respuesta a las incertidumbres desatadas en torno a lo humano y sus quimeras después del derrumbe del muro de Berlín, o a la luz de sucesos tan terribles como el 11-S. El retorno al miedo, a través de la memoria, en tiempos de la omnipresencia del terror(ismo) es, hasta cierto punto, coherente con el espíritu de la época.

La memoria del miedo, en especial cuando este es consecuencia de un episodio bélico - y su expresión cobra cuerpo en los relatos en torno al mismo-,

2. La muestra se conformó, finalmente, con 31 informantes formales (la mayoría de ellos residentes habituales de la zona objeto de estudio en la actualidad, pero también otros que residieron allí en el periodo al que se referían los hechos narrados). La red de informantes clave, 17 en total, fue entrevistada en varias ocasiones (con un total de 47 entrevistas). Con algunos de ellos, además de la grabación de las sesiones consistentes en la realización de entrevistas semiestructuradas, se llevaron a cabo otras sesiones de recogida de información a partir de la revisión conjunta de correspondencia personal o archivos fotográficos familiares y visitas a lugares de la memoria comunitaria (trincheras, campamentos de los maquis, etc.). 
no puede disociarse, en primer lugar, de la percepción del riesgo inherentemente ligado a la vivencia de toda guerra. Bauman (2007: 12) menciona que los peligros que se temen y, consecuentemente, los miedos derivativos que aquellos causan, pueden ser de tres clases: los que amenazan al cuerpo y a las propiedades personales, los que amenazan a la duración y a la fiabilidad del orden social del que depende la seguridad del medio de vida (el empleo, la producción agrícola) y, finalmente, los que amenazan el lugar de la persona en el mundo (su posición en la jerarquía social, sus creencias religiosas, su identidad étnica o de género, etc.).

Estas tres dimensiones del peligro, como veremos, atraviesan las narrativas del miedo en torno a la Guerra Civil española de los informantes, y toma forma en el riesgo de la muerte y del hambre, de la pérdida de seres y bienes, pero también en la amenaza destructiva de tradiciones y costumbres profundamente arraigadas en el imaginario y en las prácticas cotidianas de estas comunidades rurales. Y es que, en su configuración, como en todas las memorias, la del miedo también supone un ejercicio de síntesis, de eliminación de lo colateral o residual y de permanencia de ciertos núcleos duros de hechos y sentidos, en una labor de concentración o simplificación eficiente, como resultado del cual solamente queda aquello que, desde la perspectiva del sujeto, resulta más esencial y significativo.

$\mathrm{Al}$ margen de las delimitaciones conceptuales de los actores más sobresalientes que, en los últimos años, han destacado la noción de riesgo (Douglas, 1996; Beck, 1998; Alexander, 2000), nos interesa sobre todo la expresión de dichas experiencias en las narrativas de los informantes, de esta manera participamos en el ejercicio de reconceptualización, representación o recreación que se realiza por parte de los mismos. Nociones como inseguridad, vulnerabilidad, proximidad, certeza o peligros calculables emergen con profusión en esta reflexión sobre los temores y los miedos del mundo rural de hace siete décadas, contados en el marco de una sociedad que en absoluto ha dominado su miedo, sino que, más bien, ha autopropulsado otros.

En tales lecturas individuales de los sucesos en el tiempo, no interesa tanto una verdad sustentada en la exactitud —según los términos de la historia instituida o de la inasible (o imposible) noción de objetividad-, cuanto la condición de verdad que la memoria adquiere en el relato de los hechos y sus interpretaciones (res factae versus res fictae) (Lechner y Güell, 2006), con el entramado de juicios de valor en el cual se insertan, de manera natural, los acontecimientos narrados. Tomando en cuenta tales cuestiones, se debe destacar que la memoria del miedo y los recuerdos personales de la guerra y de la posguerra de los informantes tienen uno de sus ejes discursivos centrales en la distinción entre el bien y el mal, como marcas que establecen una de las principales clasificaciones de los hechos y las personas que contienen las narrativas a partir de las cuales se configura el entramado de la memoria. La diferenciación y la clasificación otorgada a cada uno de los dos grupos enfrentados en la Guerra Civil, sobre los cuales se articulan las narrativas de la memoria, aparece mediada por los valores y la cultura de los sujetos. 
Por ejemplo, en muchos casos, las banderas rojas y negras acabarían conformando el símbolo de la encarnación del mal. La calificación de cada grupo, según esta distinción central, se sustenta en el posicionamiento del sujeto que recuerda, lo cual permite lecturas distintas e incluso directamente opuestas de los grupos:

La Columna de Hierro la formaron todos los presidiarios que había en Valencia. Aquí venían con hachas, con unas polainas [...] daban miedo. Estaban en las cárceles y los metieron allí y esos hacían y deshacían lo que querían. Por aquí venían mucho, a robar y a llevarse jamones. Venían a robar y a meter miedo a la gente. Nos gritaban: «iMuchachos, que viene la Columna de Hierro!» y temblábamos. Les teníamos pánico. (Hombre, Manzanera) ${ }^{3}$

Así, la revolución, que aspiraba a reconceptualizar el bien social, acabó representando el mal para gran parte de la sociedad rural turolense de la zona, sobre todo para quienes formaban parte de un sector caracterizado por planteamientos culturales asentados en los pilares de la religiosidad popular, el valor del trabajo y el respeto a los vínculos sociales. En los recuerdos de los sujetos, se dibuja la presencia de aquellos jóvenes que arribaban mostrando insignias y consignas, repletos de banderas rojas y negras desde Castellón y Valencia, removiendo los cimientos de la tradicional vida rural:

Y la Columna de Hierro vino por aquí. ¡Hostias, si llegó! Sí, sí, de los primeros. Pero aquí lo que pasa es que luego cogían la ruta de los pueblos mejores que habían con dirección a la capital. Pero también se dieron a ver, también. ¡Ya lo creo! Se dieron a conocer y venían en camiones, con las banderas royas y negras. (Hombre, Torrijas)

Uno de los aspectos que, con mayor fuerza, se explicita como dimensión de la memoria del miedo está vinculado a la religiosidad, central en el ámbito rural. El recuerdo de la quema de las iglesias y de las reliquias veneradas a lo largo de los siglos, de generación en generación, es una escena que se repite en los relatos de los informantes de los diferentes pueblos y localidades: Manzanera, Los Cerezos, Torrijas, Camarena de la Sierra, Olba, Arcos de las Salinas:

Quemaron la iglesia al principio de la guerra. Llegaron y sacaron todos los santos a la plaza y los quemaron. ¡Un valorazo tendrían ahora! ¡No se veían las paredes, de los retablos y santos que había! También con la guerra quemaron los archivos del Ayuntamiento y se ha perdido mucho. Porque aquí había mucha cosa. [...] Y tiraron las campanas. ¡Cien arrobas pesaba la que había buena, la Santamaría!. [...] Y la iglesia, yo me acuerdo como si la viera. Todos de talla. Preciosos. Y todo lo quemaron. Porque, chica, se tapia si no quieres... Déjalo estar, pero... Tenía el coro como las catedrales con los asientos que se subían y se bajaban, el órgano, precioso, ¡que tocaba! Y me acuerdo como si

3. La identificación de los informantes, al reproducir las transcripciones literales de sus narraciones, se realizará mediante la indicación, entre paréntesis y al final del texto citado, del sexo y la comunidad en la cual habita. 
lo viera, porque tenía 12 años. Yo iba a cantar al coro. Y entrabas a la iglesia y todos los retablos preciosos, que San Antonio, que San Lorenzo, que San Isidro. Y no pudieron salvar nada. (Mujer, Manzanera)

El miedo cobra un protagonismo particular en los relatos acerca de la destrucción de los templos y los símbolos religiosos por parte de los grupos republicanos. Destaca la dimensión religiosa como uno de los ejes centrales de la conflictividad de los relatos del miedo:

Cualquiera se metía. ¿¿Cómo te metías a eso!? Yo na más me acuerdo que estaba allí y vi la primera virgen que sacaron, a la hoguera, al medio de la plaza, ahí al fuego. Me fui llorando a casa, que mi madre estaba enferma:

- ¿Qué te pasa, hija mía?

-Que han sacao los santos y los están quemando en la plaza.

$\mathrm{Y}$ así los fueron sacando hasta que quemaron todos. (Mujer, Manzanera)

En algunas poblaciones, apenas intervinieron sus habitantes. En otras, jóvenes de localidades próximas o de la propia localidad participaban en el arrasamiento de los lugares sagrados y en la destrucción y el saqueo de objetos pertenecientes a la iglesia. Pero, en general, en los relatos, se reconoce el predominio de la pasividad de los propios vecinos, que fueron incapaces de actuar para impedir lo que estaban presenciando. La parte de la población que había confiado en salir de su miseria con la llegada de la república pero que firmemente mantenía sus ideas religiosas no se identificaba con aquellos comportamientos, aunque tampoco era capaz de tomar las riendas y posicionarse. El miedo, según las narraciones, impregnaba las acciones de la mayoría de la gente, que optó por resistir y sobrevivir. Unos se excusan por su cobardía, otros se interrogan acerca de si podrían haber tomado partido y hay quienes aceptan su miedo ante el ímpetu de quienes asumían el liderazgo de estas acciones.

El miedo es eje y característica principal de los acontecimientos que se recuerdan: el miedo como presencia permanente, como protagonista de las vidas cotidianas en aquellos días:

A mi hermano lo detuvieron nada más levantarse el Movimiento, y mataron a todos sus amigos. Los metieron a la cárcel, y hoy se llevaban a cuatro, mañana se llevaban otros cuatro..., y al que se llevaban ya no volvía. Él estaba en Valencia cuando estalló el Movimiento y siempre ha sido de derechas como yo, y como toda la familia. La noche anterior de llevárselo, estaba tapao con una manta, y lo destapan y dicen: «iEste pa mañana!». Y llega la noche siguiente y dicen: «iVenga, arriba!». (Hombre, Torrijas)

Hay dos aspectos permanentes en los relatos de los individuos. En primer lugar, los cambios, los énfasis y los juicios de valor, por supuesto, responden a una lógica retrospectiva que organiza los sucesos y les da un significado según la percepción global que el sujeto tiene de su vida pasada, sus principios, valores y posicionamientos. En definitiva, los recuerdos se afincan en las experiencias vividas personalmente, de manera que el sujeto narrador es el actor y protago- 
nista del relato, pues, incluso cuando se trata de vivencias compartidas, estos hechos forman parte fundamental de su biografía individual, sobre todo en el caso de unos sucesos de tanta envergadura como un conflicto bélico.

En segundo lugar, la memoria del miedo es, ante todo, la memoria del ocultamiento, de la huida y de la muerte. Las narraciones sobre personas ocultas, generalmente familiares, huyendo de la guerra, se suceden en los relatos de los testigos, como la expresión y la reacción más generalizada ante el miedo. No hay, en los relatos, un cuestionamiento de tales actitudes, que se consideran normales en el contexto del miedo. Al contrario, son las actitudes opuestas a esta las que se destacan como singulares en tal escenario.

Así, una testigo relata:

El cura que teníamos aquí y otro que era hijo de una dueña estuvieron mucho tiempo escondidos por las casas y por la sierra, pero, como la guerra seguía, cuando pudieron, se pasaron a Teruel. Como Teruel era nacional, se quedaron allí. Y, cuando se acabó la guerra, los sacerdotes todos vinieron bien y no les pasó nada. Y uno de estos se quedó aquí. ¡Todo se tiene que pasar! (Mujer, Arcos de las Salinas)

\section{Otro testigo cuenta:}

A mi padre se lo llevaron a la guerra a hacer fortificaciones, porque tenía ya más de los 45 . Hasta los 45 fueron todos a la guerra. Yo no sé por dónde estuvieron, pero, una noche, apareció mi padre en casa y lo tuvimos oculto en casa lo menos dos meses, que luego pasó la guerra y salieron y nadie nos dijo nada... (Hombre, Los Ibáñez Altos)

Frente a estos comportamientos que no se cuestionan, sino que se consideran normales, se destaca la actitud de quienes actuaban de manera completamente opuesta:

Su hermano se venía del frente y se escondía, pero él no, era... Cuando tenían una batalla y venían las pavas a bombardearlos, nunca corría. Decía su hermano: «No vi nunca correr a mi hermano». Cuando venía la pava, se tumbaba en tierra y se ve que diría que lo que tuviera que ser para él, así sería. Así como otros se iban corriendo, él no. Y cuando aquello pasaba y no lo había matao, pues mira..., a seguir. (Hombre, Los Cerezos)

Una alternativa al ocultamiento era la huida: marchar — dejando atrás casa, propiedades $\mathrm{y}$, muchas veces, también familiares-, ante el miedo a la guerra y sus consecuencias. Sin embargo, también en este caso, no todos tomaban el camino para poner distancia personal respecto a los combates:

Los que quedaban decían: «Vámonos, que nos enganchan y nos matan!». Pero también había gente que, aunque hubiera estao metida en todo, que eran buenas personas, dijeron: «¿Vámonos? ¿Y a dónde? ¿Dónde vamos a ir con la manta al hombro por ahí con el montón de chiquillos? Pues yo me quedo aquí. 
Si me han de matar, me pongo encima de mis chiquillos y que me maten. Y si no hemos hecho nada a nadie, ¿qué?». Pero cosas de la vida. (Hombre, Paraíso)

La memoria del miedo es también, por supuesto, la memoria de la destrucción y del hambre, consecuencias directas de todo enfrentamiento bélico. Se trata, en este caso, de dos dimensiones de los discursos que no se limitan al tiempo de la guerra misma, sino que se extienden más allá de aquella, en tanto que secuelas que perduran a continuación del fin de los bombardeos y los disparos. Mientras la muerte pierde su protagonismo en los relatos de la posguerra, estas dos cuestiones permanecen e, incluso, se incrementan en las narrativas que hacen referencia al período inmediatamente posterior al fin de los hechos bélicos.

Se afirma que:

Mucha gente, al terminarse la guerra, empezó a vivir peor que mientras duró la guerra. Los unos se habían arrimao a gente que estaba en intendencia, y algo comían. Y eso pasaba porque, aunque la demás gente se muriera de hambre, los que estaban metidos ahí, esos tenían buen rancho. Por lo menos, de comer había. Que cuando se fueron aún fue peor, que entonces no había ni de los militares ni de los demás. (Hombre, Torrijas)

Y, de hecho, un indicador para medir la crudeza con que se vivió el tiempo de la guerra es el hambre padecida:

Y no pasamos mala guerra, porque, como se quedaron allí los jefes... Mi madre les guisaba y les cosía y les lavaba. Al rancho de ellos no pasamos hambre alguna. $\mathrm{Ni}$ tampoco le quitaron los machos, porque los encerró en una habitación y la cuadra la tenía vacía. Y al carro le quitó las ruedas y las envolvió en el estiércol, y como el carro estaba roto... Total, que no le quitaron nada. (Mujer, Los Cerezos)

En muchas ocasiones, los episodios de mayor miedo se encuentran intrínsecamente asociados a la obtención de alimentos en medio de la guerra y a los riesgos que, de forma inherente, acompañaban a los actos para conseguir los mismos:

Yo cuando más miedo he pasao era cuando nos tenían en la Nava a los chiquillos y de vez en cuando nos mandaban a alguno a subir pan de aquí del pueblo. Entonces lo masaban las mujeres de ocho en ocho días, pero había que bajar, porque si te falta el pan es como si te faltara todo. Y un día me mandan a mí y a mis hermanos. [...] Subiendo con el pan, donde se estrecha el riachuelo que va a Los Cerezos, jah, me cagüen!, ya te vemos que subía la aviación hacia Javalambre, que estaba ahí el frente. (Hombre, Torrijas)

En un escenario rural, por supuesto que el hambre y la subsistencia se hallan inheremente asociadas a los cultivos y a las labores del campo. A los hechos propios de la guerra, se agregaban sus consecuencias nefastas para las cosechas, una cuestión especialmente subrayada. No es extraño que un informante recuerde: 
La primera cosecha que pilló la guerra aquí, un año después o dos de empezar, fue de mala traza; ya mal trabajado. Pero el primer año que había muy buena cosecha, nada, un desastre también. La segaban los mismos militares, porque gente del pueblo, gente joven no había. El que era viejo no valía para trabajar, ¡bueno hicíamos viejos y no eran viejos! [...] Y cuadrillas de soldaos todo lo recogían, segaban el trozo que les paicía y lo llevaban a trillar allá a la provincia de Valencia, a un pueblo que le decían Titaguas. (Hombre, Torrijas)

En cierta forma, la guerra en sí misma es presentada en las narraciones como una plaga que enferma la tierra, metáfora de cultivos abandonados, escasos rendimientos y cosechas perdidas.

Terminada la guerra, el regreso aparece marcado, en los relatos de los sujetos, por el encuentro con la destrucción provocada por aquella, sobre todo en relación con dos cuestiones esenciales: otra vez, los cultivos y bienes, así como las casas, expresión física — sobre todo en el entorno rural— del hogar familiar:

Las casas nos las destejaron con la guerra; la mía no, porque había dos parientes viejos de Valbona [...]. Como algún viejo se había quedao porque no tenía nada que perder, algunas casas estaban intactas. (Hombre, Paraíso Alto)

La destrucción de la casa y la familia van de la mano en los relatos que configuran la memoria del miedo: la primera es el asiento de la segunda, y a la destrucción de aquella corresponde el desmembramiento de esta, como parte de un mismo proceso que es, al mismo tiempo, físico y emocional.

El retorno, reverso de la huida anterior, se presenta para algunos como un momento incluso más doloroso que aquella:

La ida es mala, pero la vuelta es peor. Mi padre volvió de la evacuación con cuatro hijos y mi madre. Fuimos a cambiar el dinero de la República y nos dieron once duros. Y con eso tuvimos que apañarnos los seis. (Hombre, Paraíso Alto)

El reencuentro con la desolación, la confirmación de la muerte de familiares, la certeza de las pérdidas de bienes se juntan, y ello hace de este momento un tiempo que se destaca en los recuerdos individuales de la guerra y en las narraciones que configuran la memoria colectiva.

Como se ha señalado, todos los recuerdos sobre cuya base se configura esa memoria del miedo se articulan en torno a una dualidad central, a partir de la cual se entretejen las interpretaciones, las valoraciones y los juicios de valor acerca de los hechos: el par del bien y el mal. Tomando este par como referente central, las lecturas de los recuerdos cobran sentido sobre la base de un entramado de valores y principios, muchas veces encontrados e incluso directamente contradictorios, que, efectivamente, no pueden ser comprendidos a partir de una unidicotomía estrechamente político-ideológica de izquierda y derecha.

En el posicionamiento de los individuos, se entremezclan todas las dimensiones de la realidad social —económica, política, cultural, geográfica一, sin 
admitir lecturas reduccionistas ni simplistas de los hechos. Así, por ejemplo, resulta imposible asociar la militancia republicana a una inherente postura antirreligiosa, pues, en la realidad que dibujan los recuerdos individuales configuradores de la memoria colectiva, se reconocen las muchas y complejas conflictividades que atravesaban ese período histórico.

Lo que en ciertos discursos históricos podría proponerse como irreconciliable, en tanto que recurso para dotar a los relatos de una cierta coherencia, en la memoria se presenta en toda su complejidad, sin intentar el ocultamiento de sus tensiones intrínsecas. Uno de los sujetos reconoce:

A mi abuelo le gustaba mucho ir a misa. Era republicano y los hijos fueron todos republicanos también y pelearon por la república. Aquel hombre iba a misa, sí, pero no tiene nada que ver, porque uno tiene sus creencias, ¿̇no? Y el vecino que tenía al lado..., aquellos eran del otro bando, pero eran amigos y vecinos. ¡Ahora, que cada uno de su bando! (Hombre, Manzanera)

En las narrativas que configuran la memoria del miedo, aquella dualidad del bien y el mal, que atraviesa en realidad toda la historia de las sociedades, remite en sus esencias a un entramado de valores y principios, profunda y marcadamente arraigados sobre todo en el entorno rural, que sirve de rasero de última instancia para establecer las distinciones de pertenencia grupal, aún cuando el narrador declare, de forma explícita, una cierta militancia políticoideológica. Se distingue, en fin:

Una cosa es que uno sea comunista y que sea una persona honrada y decente, porque en todas partes hay de todo. [...] ¡Bah! Eran una pandilla, más malos que malos». (Hombre, Torrijas)

\section{El miedo a la memoria: silencios y valoraciones}

Pero, a la vez que objeto (de la memoria), el miedo es, también, sujeto en las narraciones que configuran la memoria en torno a la Guerra Civil española. Una presencia que se extiende desde el momento mismo de los acontecimientos que se recuerdan hasta la actualidad. La propia decisión de alejar a los «chiquillos» del escenario bélico tiene un doble propósito de protección, tanto físico como simbólico:

Antes no se hablaba, pero después nos hemos ido dando cuenta de las cosas viendo las trincheras, las alambradas... Y lo que pasa: la gente, ignorante de lo que era la guerra. Cuando se aproximaba el frente a los Cerezos, pues ya nos sacaron del pueblo a los chavales y nos llevaron a la Nava. (Hombre, Torrijas)

Este miedo a la memoria tiene dos dimensiones de expresión claramente distinguibles en los relatos de los testigos. De una parte, es expresión de la perdurabilidad hasta nuestros días de una cultura de la sobrevigilancia profundamente enraizada - frente a la cual las respuestas fueron, justamente, las 
distintas estrategias de ocultamiento físico-simbólico puestas en práctica durante la guerra misma-; mientras, de otra, resulta una manifestación de lo que Todorov $(2000,2002)$ denomina «memoria ejemplar», esto es, la construcción de un ejemplo aleccionador, de manera tal que el pasado se convierte en principio de acción y valoración para el presente, distinguiéndola de la «memoria literal», en la cual lo narrado es preservado en su literalidad y no conduce más allá de sí mismo, por lo que el presente se somete al pasado.

Con respecto a la primera dimensión del miedo a la memoria, si el ocultamiento o la huida de la guerra es, como se ha visto, una estrategia frente al miedo, el ocultamiento de la memoria misma resulta una estrategia frente al miedo a aquella y a sus recuerdos. Un recurso, en otras palabras, para el olvido de la muerte, el dolor, el hambre, la destrucción. De hecho, en algunos relatos, se pone de manifiesto un cierto ejercicio saludable de olvido de la violencia, y se niega su existencia en un período en que ya era presencia constante y expresión de la conflictividad social en ascenso:

Con la República bien. Yo empecé antes a jornal por una peseta en las salinas, llevando al macho del ramo todo el día. Entonces eran productivas porque se cobraba poco. En los años de la República, ya se cobraba algo más de jornal. Que antes, pues nada. Y si algunos no hubieran dao tanto mal, se hubiera vivido mejor. (Hombre, Arcos de las Salinas)

Por su parte, un informante se pregunta, justificando el silencio:

Pero es que, de la guerra, ¿qué se puede contar? Yo creo que de eso cuentan poco los padres. No le voy a decir yo a un muchacho mío de diez años: «Es que fusilaron a este o al otro». (Hombre, Manzanera)

Una mujer, por su parte, confiesa:

De la guerra, mi padre jamás, ¡ni nombrarla siquiera! En mi casa, no se ha oído comentar nunca nada. Son temas muy cercanos y a lo mejor quien los ha vivido muy de cerca, como les ha ocurrido a ellos, no quieren saber nada. Mi tío murió huyendo y jamás se comenta nada. Lo mataron en Valencia, pero no fue en el frente [...]. Y, ya te digo, en mi casa nunca se habló de la guerra [...]. He oído cosas de mi tío, pero ni a mi madre ni a mi padre, ni a mi abuela. (Mujer, Torrijas)

El silencio resulta, sobre todo ante unos acontecimientos tan tremendos, un mecanismo garante de cierta calma y supervivencia social, asociado a un recurso de «olvido» intencionado sobre todo de los hechos locales, a fin de poder seguir viviendo en comunidad. Silencio y «olvido» son, así, las expresiones iniciales fundamentales de ese miedo a la memoria en torno a un hecho tan conflictivo como es la guerra.

Esto podría entenderse como una paradoja, teniendo en cuenta el carácter inherentemente colectivo, comunitario, de la memoria. La negación de la 
memoria, en este caso, sin embargo, deviene precisamente el primer mecanismo para una permanencia de lo colectivo y comunitario, esencial sobre todo en la vida cotidiana rural y en los pueblos. En realidad, la profunda, múltiple y compleja conflictividad de los hechos hace que el miedo a la memoria - continuidad de las tensiones - se convierta en recurso para la recuperación de una dimensión comunitaria desarticulada y casi destruida.

En las narraciones, los recuerdos de la guerra se entremezclan con la censura de la misma y el deseo explícito de que nunca más se repita un suceso similar, precisamente por las secuelas de largo aliento que deja en la sociedad una guerra civil:

La guerra, jaquello fue un desastre! ¡Nunca debió existir la Guerra Civil! Las guerras civiles son mucho peor que las otras, porque las otras, se acaba la guerra y no quedan rencores, y estas, aún quedan rencores de los hijos y de los... ¡ $\mathrm{Uy}$, y espérate! Eso pa generaciones. (Hombre, Torrijas)

Esa singularidad de una guerra civil justifica el silencio y el miedo a la memoria, como estrategias para - al menos - disimular los desgarramientos provocados por aquella en dos de los pilares emocionales y comunitarios fundamentales del entorno rural: la casa y el pueblo, metáforas de la familia y lo social, respectivamente. Los sujetos subrayan, de forma especial, los conflictos provocados por la guerra en estas dos instituciones:

Hermanos con hermanos. ¡Si uno estaba en un alto y el otro en otro, allí no había con quien razonar! Lo que es menester es que no vuelva. Ahora no sería igual, arderá todo de una, y mejor eso antes que matar a un hermano. (Hombre, Arcos de las Salinas)

En particular, los conflictos generados en el interior de la institución familiar se consideran la expresión más terrible de la Guerra Civil:

Es duro, hermanos con hermanos, ¡coño! Un hermano perseguido y el otro decirle: «Hijo puta». Hay casos de todos los colores. Es más que malo. (Hombre, Manzanera)

$\mathrm{Al}$ régimen del miedo sucedió, de manera inmediata, el régimen del silencio. No solo por el temor a una continuidad de los conflictos, inherente a la configuración de la memoria, sino también por la propia perdurabilidad del miedo mismo, más allá del fin de los episodios bélicos. El silencio se instaló como una doble estrategia de supervivencia, física individual y colectiva comunitaria al mismo tiempo:

¿Sabes qué pasa?, que miedo hemos pasao todos mucho, pero la gente se callaba y ya está y a vivir como cada uno podía. Veías algo que llamaba la atención y a callar. Hicieron una vez un toro embolao y había siete u ocho con unos trajes de pana nuevos, que entonces ¡ojo!, y la gente miraba y: «¿Quiénes serán estos? 
¿Serán maquis?». ¡Claro! Pero la gente se callaba. Lo sabías, pero te callabas. (Hombre, Los Cerezos)

No obstante, con el tiempo, la memoria iría emergiendo y reactivándose, de modo que los nietos han sido mucho más depositarios de una memoria que tiene un fuerte contenido moral, de lo que fueron sus propios padres:

Yo muchas cosas las sé porque lo he oído alguna vez. Es que nunca hemos hablado estas cosas; antes es que ni se podía... La gente no quería hablar de todo lo que había pasado. Ahora es cuando hay cierta libertad para hablar algo por lo menos, ¿¿no? (Hombre, Manzanera)

El seno familiar, como explican los informantes, no fue entorno propicio para las referencias explícitas a la guerra en las primeras generaciones:

Hay gente que en las casas habla más. No sé como explicarme. Por ejemplo, no hace mucho, mi tía comentó algo de mi familia que yo no sabía. Otro día, en otra casa, una tarde salió el tema y la tía A. dijo: «Pues ya te voy a decir yo por que». Es que había habido un motín en tuel pueblo [...]. Pero jaleo, pero jaleo. Y mi tía era pequeñica y se acuerda de llorar y de que a su madre..., pero lo que pasa: que hay casas que dan mucho a saber a los hijos y otras que las cosas que no son buenas las quieres tapar. [...] Algunas te las han contao los padres y otras se han tapao o no se han conocido. (Mujer, Arcos de las Salinas)

Más adelante, el escenario familiar se fue convirtiendo en el canal más adecuado para la configuración de la memoria factual, mientras que la valorativa encontró espacio en el contexto comunitario. La dimensión más significativa de la memoria valorativa que se ha ido configurando en el espacio comunitario está constituida por la actitud y los juicios de valor negativos sobre la política que se han ido articulando como parte de la recuperación de la memoria del tiempo de la guerra. Unos juicios de valor que estarían asociados a unas actitudes marcadas por cierto distanciamiento o no compromiso, al menos explícito, con respecto a lo político, que se ha ido transmitiendo de una generación a otra:

Mi padre nunca se ha metido en política y eso nos enseñó. Hubo un referéndum cuando Franco, y mi padre diría, pues lo que tenían que decir todos. Lo que tuvieran que decir. (Hombre, Manzanera)

Uno de los sujetos compara la calidad de la política de aquellos tiempos y la actual:

Entonces había mucha política. Al baile no iban todos juntos, había un baile $p a$ unos y un baile $p a$ otros. Todos juntos, no. La música no sonaba bien (ríe). (Hombre, Los Cerezos)

Pero no solo se trataba del concepto y los principios de lo político en general, sino también de la forma en que funcionaban sus mecanismos y sus estructuras: 
Ahora dicen de las elecciones, pero ¡entonces sí había jaleo! Iban por las aldeas y las masías buscando los votos. ¡A buscar votos todos! Mi madre me decía que, cuando iban a ser las elecciones, a la derecha le faltaba un voto, y se cantaba: «Joaquín de Lino al Moral, / Ernesto al barranco el Agua, / pero no han podido encontrar / el voto que les faltaba». Ahora dicen que hay política, pero entonces había más política que ahora. Eso era de cuando la República, antes de la guerra. Y los de derechas buscaban los votos por todos sitios. (Hombre, Los Cerezos)

La política es definida por algunos como «un embuste», y la conceptualización asociada a la clase política se encuentra matizada por las siguientes apreciaciones: «Son personas que se entrometen en todo», que «les gusta estar enterados de todo». "Ser politiquillo» supone también "tener facilidad para hablar y enterarse de las cosas» y buscar el beneficio particular. En concreto, se considera que:

Al que le toque, que haga las cosas; $y$, si no, tienes la prueba ahora con nuestros políticos. Si tiene España dos o tres problemas, como es el terrorismo, como es el paro, estas cosas... ¡ Coñe, tu, el que tiene obligación, que les hinque el diente! Pues no, no hacen más que echarse los trastos los unos a los otros y punto. Así no hacen nada. Se andará, pero yo no sé hasta dónde, y así no, así no se puede. (Hombre, Olba)

Tales valoraciones remiten, de manera directa, a la particular conflictividad inherente a la recuperación de la memoria del miedo desde los discursos históricos oficiales y, recurriendo a las nociones de Maffesoli (1993), el miedo (de lo) instituido a la memoria instituyente. A la encrucijada entre el pasado mítico y el pasado real que, como plantea Huyssen (2002), le resulta inherente a toda política de la memoria - tanto por los efectos de realidad que pueden emerger de lo mítico, como dada la posible mitologización de lo real—, se agrega el cuestionamiento que la memoria instituyente supone para lo (político) instituido.

Ciertas políticas de la memoria, en respuesta a este miedo (de lo) instituido a la memoria instituyente, proponen una recuperación de aquella basada en una jerarquización de las memorias desde el discurso público (Huyssen, 2002) que intentaría, en tal sentido, eliminar - $\mathrm{u}$ ocultar- las memorias inherentes en conflicto. Esta segunda dimensión del miedo a la memoria, estrechamente ligada a una distribución de tareas según la cual la memoria factual —relacionada con los hechos más cercanos, con lo directamente vivenciado por los individuos y la comunidad - tiene su principal escenario en el ámbito familiar, mientras la memoria valorativa halla su espacio en lo comunitario, configura entonces un escenario de mayor conflictividad entre la memoria instituyente valorativa y los discursos históricos desde el poder. Esta dimensión del miedo a la memoria abre, sin duda, toda una línea de indagación en torno a la inherente resistencia de la memoria a la institucionalización y su conflictividad con lo instituido, ligados, respectivamente, a una singularidad y 
una universalidad en las cuales no tiene cabida la particularidad — esto es, su diversidad y recreación constante, inherentes a los procesos de configuración de la memoria colectiva a partir de las narrativas resultantes de los recuerdos individuales.

\section{Conclusiones: la memoria del miedo y el miedo a la memoria}

La memoria de la guerra, que se configura a partir de los recuerdos individuales de los sujetos, aparece atravesada por un eje central, el miedo, objeto y sujeto de las narraciones a partir de las cuales se articulan esos recuerdos individuales con la memoria colectiva.

En los relatos de los sujetos sobre la Guerra Civil española, son recurrentes varios ejes temáticos: en primer lugar, la muerte, la expresión más terrible de un conflicto bélico. Junto a este, los distintos comportamientos de los individuos frente a un acontecimiento de tal naturaleza, sobre todo el ocultamiento y la huida. El primero, resulta una respuesta que se considera normal ante el miedo a la muerte que acompaña a toda guerra; la segunda, también estrategia de alejamiento del dolor y los peligros asociados a toda contienda armada, aunque con el agregado que implica la separación de la tierra, la comunidad y la casa, el desarraigo y la ruptura asociados a la partida en este caso, mucho más marcado dado el profundo sentido de pertenencia que caracteriza la relación con el territorio en el ámbito rural, la vinculación a lo largo de generaciones y generaciones a un cierto pueblo y a su geografía.

$\mathrm{Al}$ mismo tiempo que los comportamientos frente al miedo, las consecuencias de la guerra también son ejes recurrentes en las narrativas que configuran la memoria del miedo, sobre todo la muerte, el hambre y la destrucción. La muerte es, como corresponde tratándose de relatos sobre un acontecimiento bélico, la temática central en muchos de los testimonios de los sujetos. El hambre y, estrechamente asociada a ella, la destrucción de pueblos y de los bienes materiales también resultan dos elementos frecuentes en los relatos. De hecho, habitualmente, son considerados como indicadores de los niveles con que se padeció la guerra. Quienes tuvieron alternativas para sortear el hambre y cuyas casas y propiedades no fueron destruidas o saqueadas resaltan estas circunstancias en sus testimonios, como muestra de haber evitado dos de las principales consecuencias de la guerra.

Pero mientras la memoria de la muerte se concentra en el período a lo largo del cual duraron las acciones bélicas — aunque sin limitarse a este-, el hambre y la destrucción aparecen asociadas, en muchos casos, más al período posbélico, como las dos consecuencias materiales que perduraron, con más fuerza y por más tiempo, más allá del fin de las batallas. Tratándose de un entorno rural, la destrucción se asocia, de forma inherente, a los cultivos y a las cosechas, con sus secuelas de hambre. La guerra es considerada, desde este escenario, una plaga que perdura más allá del cese de los disparos y los bombardeos. 
Pero la guerra no solo tiene consecuencias materiales, sino también emocionales. Precisamente es en relación con estas que, en la propia memoria del miedo, se presenta, como elemento de los relatos configuradores, el miedo a la memoria. Un temor a la memoria estrechamente ligado a las particularidades de una guerra civil y que cobra cuerpo en dos expresiones fundamentales: el silencio y los juicios de valor sobre la política y los políticos.

En las valoraciones acerca de la guerra, se destacan sus particulares consecuencias, debido a su condición de guerra civil, para lo colectivo. El desmembramiento de lo colectivo - en particular, de dos de sus pilares fundamentales en lo rural, la familia y la comunidad (el pueblo) - es especialmente marcado en este caso y constituye una de las cuestiones que se consideran más terribles como secuela del conflicto. De ahí se deriva que si, durante la guerra, se trataba de ocultarse de la misma como estrategia de supervivencia individual, después de la guerra, la cuestión era ocultarla como parte de una estrategia de recuperación o reconstrucción de lo colectivo, o al menos lo poco que había quedado del mismo.

El silencio se convierte, desde esta perspectiva, en recurso para evitar una renovación de aquellas conflictividades que han sido destructoras de los sentidos colectivos y, en cierta medida, ir rescatando los tejidos articuladores que hacen posible el encuentro, ya sea familiar o comunitario. Pudiera parecer una paradoja que, en el ámbito rural, con la importancia que en el mismo tiene la oralidad como mecanismo de configuración de lo colectivo y lo público, el silencio se presente como la estrategia más oportuna para salvar (o recuperar) lo comunitario. Sin embargo, se trata justamente de silenciar una dimensión discursiva de la memoria que, por su contenido especialmente conflictivo, podría (re)generar tensiones que afectaran, de manera profunda, a los procesos de configuración de lo comunitario. Este silencio temático se considera, por consiguiente, condición de posibilidad para superar la profunda conflictividad de los episodios de la Guerra Civil española y hacer posible la recuperación de los sentidos de lo comunitario afectados.

Sin embargo, este silencio resulta, en última instancia, un imposible, pues la propia profundidad del conflicto que caracteriza a los hechos implica la necesidad de articular narrativas configuradoras de la memoria colectiva en torno a los mismos. En la superación del silencio y la articulación de narrativas de la memoria del miedo, debe tener lugar, sin embargo, una distribución de escenarios y temáticas que facilite ese ejercicio imprescindible de configuración de la memoria colectiva del miedo, pero tratando de evitar al máximo las posibles reemergencias de conflictividades.

En tal sentido, el escenario comunitario queda reservado para una recuperación de la dimensión valorativa de la memoria del miedo, lo cual permite ubicarse en niveles más generales, con juicios de valor sobre la política y los politicos desde un punto de vista, si se quiere, más general. Es como parte de esta dimensión de la memoria colectiva que se explicitan las valoraciones negativas acerca de la política y de los políticos que atraviesan los relatos individuales. Por su parte, la recuperación de la dimensión factual de la memoria del miedo 
queda, todavía hoy, restringida al ámbito familiar, con lo cual se evita una recuperación pública de hechos demasiado cercanos, que podrían provocar el resurgimiento de conflictividades en lo local —el pueblo- y que incidirían negativamente sobre las condiciones de posibilidad de regeneración del tejido comunitario, tan importante en este escenario.

\section{Referencias bibliográficas}

Aguilar Fernández, Paloma (1996). Memoria y olvido de la Guerra Civil española. Madrid: Alianza Editorial.

AleXANDER, Jeffrey (2000). «Ciencia social y salvación: Sociedad del riesgo como discurso mítico». En: Sociología cultural: Formas de clasificación de las sociedades complejas. México: Anthropos/FLACSO, 1-30.

ANDERSON, Benedict (1993). Comunidades imaginadas: Reflexiones sobre el origen y la difusión del nacionalismo. México: Fondo de Cultura Económica.

BAUMAN, Zygmunt (2007). Miedo líquido: La sociedad contemporánea y sus temores. Barcelona: Paidós.

BECK, Ulrich (1998). La sociedad del riesgo: hacia una nueva modernidad. Barcelona: Paidós.

BeEvor, Antony (2009). La Guerra Civil española. Barcelona: Crítica.

BILBAO, Andrés (2007). Individuo y orden social: La emergencia del individuo y la transición a la sociología. Madrid: Sequitur.

BorTo, María Eugenia y DE LA Cruz, Eduardo Maximiliano (2000). «La importancia de la oralidad en la cultura contemporánea. El caso de "El Caldero de los cuentos en Córdoba"». Revista Latina de Comunicación Social [en línea], 35 (3). Número extra: «La comunicación social en Argentina». <http://www.ull.es/publicaciones/ latina/argentina2000/21boito.htm>.

DOUGLAS, Mary (1996). La aceptabilidad del riesgo según las ciencias sociales. Barcelona: Paidós Ibérica.

FouCAUlt, Michel (1979). Microfisica del poder. Madrid: La Piqueta.

FRASER, Ronald (2005). Recuérdalo tú y recuérdalo a otros: Historia oral de la Guerra Civil española. Madrid: Planeta-De Agostini.

HalbWaChS, Maurice (1968). La mémoire collective. París: Presses Universitaires de France.

HUYSSEN, Andreas (2002). En busca del futuro perdido: Cultura y memoria en tiempos de globalización. México: Fondo de Cultura Económica.

IBÁŃEZ, Jesús (1988). «Relatividad física y cuántica sacuden las ciencias sociales. Tendencias científicas y sociales». En: IBÁÑEZ, Jesús (coord.). Nuevos avances en la investigación social I. Barcelona: Proyecto A Ediciones.

LECHNER, Norbert y GÜELL, Pedro (2006). «Construcción social de las memorias en la transición chilena». En: JeLIn, Elizabeth y Kaufan, Susana (comps.). Subjetividad $y$ figuras de la memoria. Buenos Aires: Siglo XXI.

LuENGO, Ana (2004). La encrucijada de la memoria: La memoria colectiva de la Guerra Civil española en la novela contemporánea. Berlín: Tranvía-Verlag Walter Frey.

MafFesoli, Michel (1993). El conocimiento ordinario: Compendio de Sociología. México: Fondo de Cultura Económica.

- (2009). Iconologias: Nuestras idolatri@s postmodernas. Madrid: Península.

MANGINI GonZÁLEZ, Shirley (1997). Recuerdos de la resistencia: La voz de las mujeres de la Guerra Civil española. Madrid: Península. 
Sanz Hernández, Alexia y Ramos Martín, Manuel (2009). Peligro, riesgo y guerra. Memorias del miedo. Zaragoza: Departamento de Educación, Cultura y Deporte, Gobierno de Aragón.

Sotolongo Codina, Pedro Luis y Delgado Díaz, Carlos Jesús (2006). «La intersubjetividad social, las estructuras sociales objetivadas y las subjetividades sociales individuales». En: Sotolongo Codina, Pedro Luis y Delgado Díaz, Carlos Jesús (comps.). La revolución contemporánea del saber y la complejidad social: Hacia unas ciencias sociales de nuevo tipo. Buenos Aires: Consejo Latinoamericano de Ciencias Sociales, 131-164.

Todorov, Tzvetan (2000). Los abusos de la memoria. Madrid: Paidos.

- (2002). Memoria del mal, tentación del bien. Madrid: Península.

Traverso, E. (2007). El pasado, instrucciones de uso: Historia, memoria, política. Barcelona: Marcial Pons. 